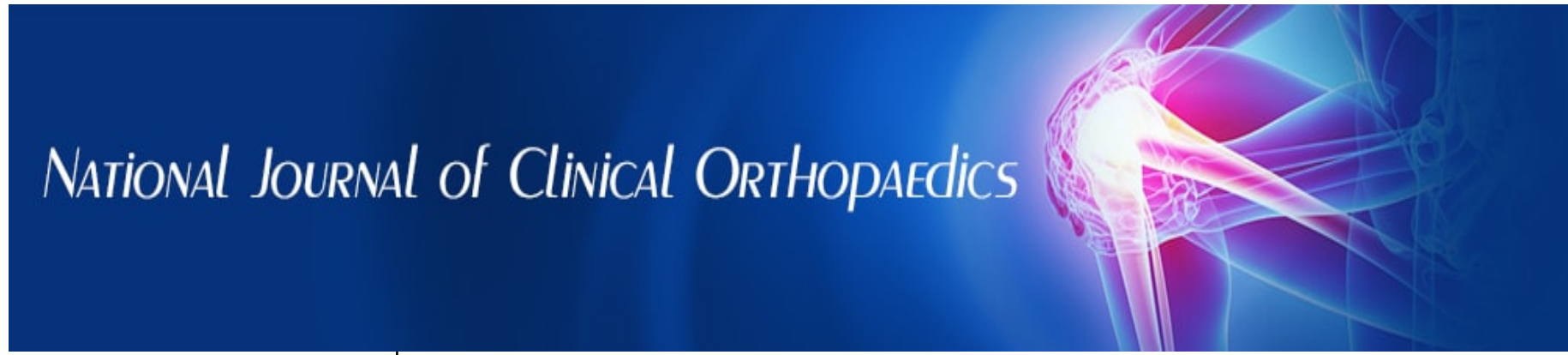

ISSN (P): 2521-3466

ISSN (E): 2521-3474

(C) Clinical Orthopaedics

www.orthoresearchjournal.com

2021; 5(3): 13-15

Received: 27-04-2021

Accepted: 08-06-2021

Savvina Theologou

Department of Physiotherapy,

Faculty of Health Sciences

International Hellenic University

Alexander Campus P.O. Box 141,

57400 Sindos, Thessaloniki, Greece

Dimitrios Lytras

Department of Physiotherapy,

Faculty of Health Sciences

International Hellenic University

Alexander Campus P.O. Box 141,

57400 Sindos, Thessaloniki, Greece

Paris Iakovidis

Department of Physiotherapy,

Faculty of Health Sciences

International Hellenic University

Alexander Campus P.O. Box 141,

57400 Sindos, Thessaloniki, Greece

Anastasios Kottaras

Department of Physiotherapy,

Faculty of Health Sciences

International Hellenic University

Alexander Campus P.O. Box 141,

57400 Sindos, Thessaloniki, Greece

Anna Giannakoudaki

Department of Nutritional Sciences

and Dietetics, Faculty of Health

Sciences International Hellenic

University - Alexander Campus

P.O. Box 141, 57400 Sindos,

Thessaloniki, Greece

Konstantinos Moutaftsis

Department of Physiotherapy,

Faculty of Health Sciences

International Hellenic University

Alexander Campus P.O. Box 141,

57400 Sindos, Thessaloniki, Greece

Corresponding Author:

Savvina Theologou

Department of Physiotherapy,

Faculty of Health Sciences

International Hellenic University

Alexander Campus P.O. Box 141,

57400 Sindos, Thessaloniki, Greece

\section{The efficacy of transcutaneous electrical neuro stimulation (TENS) in reducing pain in patients with cervical syndrome}

\section{Savvina Theologou, Dimitrios Lytras, Paris Iakovidis, Anastasios Kottaras, Anna Giannakoudaki and Konstantinos Moutaftsis}

DOI: https://doi.org/10.33545/orthor.2021.v5.i3a.284

\section{Abstract}

Background: The cervical syndrome is a set of different symptoms that are located in and around the neck area. Physiotherapy and specifically electrotherapy through can help relieve pain in patients with cervical syndrome.

Aim: The aim of this review is to highlight, through recent articles, the efficacy of TENS in reducing pain in patients with cervical syndrome.

Method: The Google Scholar and PubMed databases were searched in English and Greek with the following keywords: cervical syndrome, Transcutaneous Electrical Nerve Stimulation, TENS, physiotherapeutic rehabilitation. Clinical studies and systematic reviews were included in the review.

Results: In total 10 sources were included in this review, of which seven were clinical trials and three were reviews.

Discussion-Conclusions: Applying TENS, either low-frequency or high-frequency, for 30 minutes with three sessions per week and duration of treatment varying from three to six weeks can effectively help reduce pain in patients with acute or chronic cervical syndrome.

Keywords: Cervical syndrome, transcutaneous electrical nerve stimulation, TENS, physiotherapeutic rehabilitation

\section{Introduction}

Spinal disorders are conditions that affect the cervical spine, which consists of the top seven vertebrae that surround and protect the spinal cord. The cervical spine contains many different anatomical compositions, including muscles, bones, ligaments and joints that have nerve endings and can detect adverse problems when they occur. These nerves provide muscle control and sensation to the skull and arms, and flexibility and movement to the body ${ }^{[1]}$. The cervical syndrome is a set of symptoms, the cause of which is located mainly in the occipital, cervical and shoulder area. However, it can also be found in other areas of the body, such as the head, chest and spine ${ }^{[2,3]}$.

Factors associated with the development or persistence of cervical syndrome are psychopathology, low job satisfaction, occupation or poor working environment, female gender, concomitant back pain or other rheumatic conditions, poor coping skills, trauma or previous neck injury, poor health, sedentary lifestyle, smoking and headache ${ }^{[4]}$.

Significant heterogeneity is observed between epidemiological studies of cervical syndrome, which makes it difficult to compare or collect data from different studies. The estimated incidence of neck pain from the available studies ranges between $10.4 \%$ and $21.3 \%$ with a higher incidence in office and computer workers. However, there are studies that show that between $33 \%$ and $65 \%$ of people have recovered from an episode of neck pain in one year ${ }^{[5]}$. Most cases go through an episodic incidence in the life of an individual and, therefore, relapses are common. The overall prevalence of neck pain in the general population ranges between $0.4 \%$ and $86.8 \%$ with the annual prevalence ranging from $4.8 \%$ to $79.5 \%$. Prevalence is generally higher in women, in high-income countries than in low- and middle-income countries, and in urban areas than in rural areas. 
Furthermore, most studies show an increased risk of neck pain up to the age group 35-49 years, after which the risk begins to decrease ${ }^{[5,6]}$.

Physiotherapy plays an important role in treating neck pain. Transcutaneous Electrical Nerve Stimulation (TENS) is widely used in healthcare because it is cheap, safe and can be administered even by patients themselves ${ }^{[6,7]}$. Moreover, TENS, as a physiotherapy device, has been compared for its effectiveness with other analgesic interventions and according to clinical research can relieve pain even in patients with cervical syndrome ${ }^{[8]}$. The aim of this review is to describe the efficacy of TENS in reducing pain in people with cervical syndrome.

\section{Methods}

The Google Scholar and PubMed were searched in English and Greek with the following keywords: cervical syndrome, Transcutaneous Electrical Nerve Stimulation, TENS, physiotherapeutic rehabilitation.

\section{Results}

This review included clinical trials and systematic reviews. Below are the main conclusions of the articles included in this review.

\section{Literature review}

Paolucci et al. ${ }^{[9]}$ performed an umbrella review of systematic reviews to demonstrate the efficacy of TENS in patients with acute or chronic cervical syndrome. The studies they included used as an intervention method either low-frequency TENS (1$5 \mathrm{~Hz})$ or high-frequency TENS $(60-100 \mathrm{~Hz})$, for three weeks with treatment time varying from 10 to 30 minutes. The results of their review showed that TENS helps reduce pain regardless of the progress of the condition. However, further studies are needed to support this view.

In their trial, Debre et al. ${ }^{[10]}$ investigated the effect of physiotherapy in patients with cervical myofascial pain. The study involved 90 participants aged 18-30 years, who were divided into three groups. The first group received TENS treatment, the second received exercises and the third group received friction massage. Treatment was performed three times a week for four weeks. Pain with the Visual Analog Scale (VAS) and the Neck Pain and Disability Index and level of depression with Beck's Depression Inventory was evaluated before and after intervention. This study results revealed no significant differences between the groups. All three groups showed a reduction in pain. The authors conclude that friction massage, exercise and TENS are equally effective interventions to manage neck pain.

Yesil et al. [11] investigated whether TENS and interferential current (IFC) increase the efficacy of neck stabilization exercises on pain, disability, mood and quality of life for chronic neck pain, either individually or in combination. A total of 81 patients with chronic cervical syndrome were divided into three groups (TENS group, IFC group and combination group). The intervention had a duration of weeks with 6- and 12-month follow ups. Pain intensity with the VAS, cervical range restriction (range of motion) with a goniometer, quality of life with the 36-item Short Form questionnaire, depression with the Beck depression inventory scale, disability with the Neck Pain and Disability Index and the intake of analgesics were evaluated before treatment and at the 6- and 12-week follow-ups. The findings of this study suggest that all three groups equally benefited from their respective treatment.

In another study, Martimbianco et al. ${ }^{[12]}$ also attempted to evaluate the efficacy of TENS in the treatment of cervical syndrome. Their review included randomized controlled trials involving adult patients with cervical syndrome (duration longer than 12 weeks). The main intervention used was TENS (60$100 \mathrm{~Hz}$ ) with a session duration of 20-60 minutes. The results showed a reduction in pain in patients with cervical syndrome, however, further studies are needed as the data found are of low certainty for the efficacy of TENS.

Regarding manual techniques, Khan et al. ${ }^{[13]}$ studied the effects of massage and TENS in patients with cervical syndrome. There were 40 participants, who were randomly divided into two equal groups. Group A received $40-70 \mathrm{~Hz}$ TENS treatment for 20 minutes and group $\mathrm{B}$ received massage for 30 minutes. Both groups had three sessions per week and a total of 18 sessions over a period of six weeks. At the end of six weeks, the results showed a significant reduction in pain but the efficacy of TENS was less compared to that of massage.

Chiu et al. ${ }^{[14]}$ evaluated the efficacy of TENS compared to exercise and control in 218 individuals with chronic neck pain. The intervention had a duration of six weeks with a 6-month follow-up. Pain with the verbal numeric pain scale and the Northwick Park Neck Pain Questionnaire and isometric neck muscle strength were assessed before and after the intervention. The results of this study show that both TENS and exercise are equally effective in reducing pain in individuals with chronic neck pain.

In a different study, Park et al. ${ }^{[15]}$ studied the effect of TENS in patients with cervical syndrome who have undergone thyroidectomy. Their study involved 100 patients: 50 in the group receiving $100 \mathrm{~Hz}$ TENS therapy for 30 minutes and 50 in the control group. An 11-point scale was used to evaluate the interventions 30 minutes, six hours, 24 hours and 48 hours after surgery. The findings showed lower values on the scale and concluded that TENS can be used to reduce pain in patients with cervical syndrome after thyroidectomy.

Another study that concerned manual therapy was that of Escortell-Mayor et al. ${ }^{[16]}$, who studied the efficacy of mobilization techniques and TENS in reducing pain in patients with cervical syndrome. The study involved 90 patients, of whom 47 received mobilization techniques for 30 minutes and 43 received $80 \mathrm{~Hz}$ TENS for 30 minutes. The results were evaluated with the VAS, before and six months after the intervention. Therefore, no differences were observed between the two groups as both methods were analyzed in the short-term and found to help reduce pain.

Lastly, Maayah \& Al-Jarrah ${ }^{[17]}$ evaluated TENS as a treatment for cervical syndrome due to musculoskeletal disorders. The participants were 30 people with cervical syndrome, who were randomly divided into two groups and were given either treatment with $4-8 \mathrm{~Hz}$ or placebo therapy. Each group received one session for one hour and the results were evaluated before and after the intervention with the myometer. The researchers found that TENS is an effective treatment for cervical pain due to musculoskeletal disorders. However, TENS showed pain relief in people with mild pain and not severe symptoms.

\section{Discussion-Results}

The results of this review show that TENS is used in painful conditions in which pain is the most common cause that directs patients to this therapeutic intervention. It is a modern form of analgesic electric current for pain management and is widely used by health professionals and especially by physiotherapists. Although the clinical efficacy of TENS is questionable for the complete recovery of pain, the current literature shows that 
TENS contributes significantly to pain relief even in patients with cervical syndrome.

Applying TENS to a rehabilitation program in adult patients with cervical syndrome can help relieve pain. Specifically, the administration of TENS - either low-frequency $1-5 \mathrm{~Hz}$ or highfrequency $60-100 \mathrm{~Hz}$, for 30 minutes of intervention, about three sessions per week, treatment period of 3-6 weeks - and the use of the VAS for pain assessment before and after each treatment can offer the best results in reducing pain in patients who experience mild or even severe symptoms. Therefore, TENS in combination with the VAS can improve pain in patients with cervical syndrome regardless of whether they are acute or chronic.

\section{References}

1. Cannon DE, Dillingham TR, Miao H, Andary MT, Pezzin LE. Musculoskeletal Disorders in Referrals for Suspected Cervical Radiculopathy. Arch Phys Med Rehabil 2007;88(10):1256-1259. doi:10.1016/j.apmr.2007.07.010

2. Lytras D, Sykaras E, Christoulas K, Myrogiannis I, Kellis E. Effects of an integrated neuromuscular inhibition technique program on neck muscle strength and endurance in individuals with chronic mechanical neck pain. J Body w Mov Ther 2019;23(3):643-651. doi:10.1016/j.jbmt.2019.01.004

3. Lytras DE, Sykaras EI, Christoulas KI, Myrogiannis IS, Kellis E. Effects of Exercise and an Integrated Neuromuscular Inhibition Technique Program in the Management of Chronic Mechanical Neck Pain: A Randomized Controlled Trial. J Manipulative Physiol Ther 2020;43(2):100-113. doi:10.1016/j.jmpt.2019.03.011

4. Cohen SP. Epidemiology, diagnosis, and treatment of neck pain. Mayo Clin Proc 2015;90(2):284-299. doi:10.1016/j.mayocp.2014.09.008

5. Hoy DG, Protani M, De R, Buchbinder R. The epidemiology of neck pain. Best Pract Res Clin Rheumatol 2010;24(6):783-792. doi:10.1016/j.berh.2011.01.019

6. Lytras D, Myrogiannis I, Sykaras E. The efficacy of manual therapy and therapeutic exercise in patients with chronic neck pain: A narrative review. Int J Phys Educ Sport Heal. 2018;5(1):32-36. www.kheljournal.com.

7. Lytras D, Iakovidis $\mathrm{P}$, Kottaras A, Tsimerakis AF, Myrogiannis IS. A review on interferential current analgesic effects in individuals with chronic low back pain. Int J Phys Educ Sport Heal 2021;8(3):01-04. doi:10.22271/kheljournal.2021.v8.i3a.2051

8. Jones I, Johnson MI. Transcutaneous electrical nerve stimulation. Contin Educ Anaesthesia, Crit Care Pain 2009;9(4):130-135. doi:10.1093/bjaceaccp/mkp021

9. Paolucci T, Agostini F, Paoloni M et al. Efficacy of TENS in cervical pain syndromes: An umbrella review of systematic reviews. Appl Sci 2021;11(8). doi:10.3390/app11083423

10. Debre E, Alptekin K, Alptekin JÖ, Giritharan A. Study on the effect of TENS, exercise and friction massage on the masseter regarding masseter-derived cervical myofascial pain. Adv Rehabil 2020;34(2):1-9. doi:10.5114/areh.2020.94316

11. Yesil H, Hepguler S, Dundar U, Taravati S, Isleten B. Does the Use of Electrotherapies Increase the Effectiveness of Neck Stabilization Exercises for Improving Pain, Disability, Mood, and Quality of Life in Chronic Neck Pain? 2018, 43. doi:10.1097/BRS.0000000000002663

12. Martimbianco ALC, Porfírio GJM, Pacheco RL, Torloni
MR, Riera R. Transcutaneous electrical nerve stimulation (TENS) for chronic neck pain. Cochrane Database Syst Rev 2019, (12). doi:10.1002/14651858.CD011927.pub2

13. Atif Khan M, Asif M, Hira Islam R, Azhar Mughal M, Aftab K, Riaz Baig Chughtai M. Effects of Acupressure \& TENS along with Hot Pack in Neck Pain. J Phys Fitness, Med Treat Sport 2017;1(1):1-5. doi:10.19080/jpfmts.2017.01.555555

14. Chiu TTW, Hui-Chan CWY, Cheing G. A randomized clinical trial of TENS and exercise for patients with chronic neck pain. Clin Rehabil 2005;19(8):850-860. doi:10.1191/0269215505cr920oa

15. Park C, Choi JB, Lee YS et al. The effect of intra-operative transcutaneous electrical nerve stimulation on posterior neck pain following thyroidectomy. Anaesthesia 2015;70(4):434-439. doi:10.1111/anae.12933

16. Escortell-Mayor E, Riesgo-Fuertes R, Garrido-Elustondo S et al. Primary care randomized clinical trial: Manual therapy effectiveness in comparison with TENS in patients with neck pain. Man Ther 2011;16(1):66-73. doi:10.1016/j.math.2010.07.003

17. Maayah M, Al-Jarrah M. Evaluation of Transcutaneous Electrical Nerve Stimulation as a Treatment of Neck Pain due to Musculoskeletal Disorders. J Clin Med Res 2010;2(3):127-136. doi:10.4021/jocmr2010.06.370e. 